\title{
Assessing the impact of Connect 2 Care on the residential stability of homeless and vulnerably housed clients
}

Kyle Dewsnap ${ }^{1}$, Hasham Kamran ${ }^{1}$, Kimberly Rondeau ${ }^{1}$, Alicia Polachek ${ }^{1}$, Gabriel Fabreau², Kerry McBrien ${ }^{2}$

${ }^{1}$ W21C Research and Innovation Centre, Cumming School of Medicine, University of Calgary, Calgary, Alberta, Canada.

${ }^{2}$ Community Health Sciences, Cumming School of Medicine, University of Calgary, Calgary, Alberta, Canada.

\section{Abstract}

Certain kinds of housing instability, such as foreclosure and homelessness, have been associated with poorer physical and mental health. The Connect 2 Care (C2C) program targets medically complex individuals who are unstably housed, primarily aimed at reducing acute care utilization and connecting clients to appropriate community-based care. However, because housing status is a fundamental determinant of health, the team also assists clients in finding permanent housing. As the $\mathrm{C} 2 \mathrm{C}$ program aims to improve the health of its clients, we hope that this intervention positively impacts the housing stability of clients. The objective is to determine whether the $\mathrm{C} 2 \mathrm{C}$ program is effective in reducing factors of housing instability, such as the frequency of housing moves made, and time spent in unstable housing (such as shelters or sleeping outside). C2C clients were asked to participate in 6- and 12- month follow-up surveys with a member of the research team. During both surveys, participants were prompted to describe their housing history using the Residential Time-Line Follow-Back (rTLFB) inventory. Starting at six months prior to their intake into $\mathrm{C} 2 \mathrm{C}$, participants created a twelve- to eighteenmonth timeline that detailed their residential locations and number of housing transitions. Location descriptions provided by clients were categorized as stable, temporary, institutional, or literal homelessness. The number of housing transitions and the proportion of time spent in each housing category were then calculated for each individual. Changes in proportion of time spent over three unique time periods were evaluated using Wilcoxon's paired rank test with Holm's multiplicity correction. Since September 2018, housing data was collected from 100 unique clients. In comparing the six months preceding $\mathrm{C} 2 \mathrm{C}$ intake with the six-to-twelve months after C2C intake, significant reductions in the amount of time spent in literal homelessness $(p<$ $0.001)$ and reductions in the number of housing changes $(p=0.014)$ were observed. Housing stability for $\mathrm{C} 2 \mathrm{C}$ clients improved after enrolment in the program. This study was potentially limited by incomplete sampling of the C2C population. Based on our findings, further research should be conducted in evaluating the relationship between increases in housing stability and increases of health status.

\section{Key words}

Homelessness, unstable housing, social determinants of health, health navigation, health outreach.

Cite as: Dewsnap K., Kamran H., Rondeau K., Polachek A., Fabreau G., McBrien K. 2019. Assessing the impact of Connect 2 Care on the residential stability of homeless and vulnerably housed clients. Alberta Academic Review, Vol 2 (3) 17, CASCH Special Issue (not peer-reviewed), DOI: 10.29173/aar107. 